\title{
DIVERSIDAD FLORÍSTICA EN UN PAISAJE RURAL DEL PIEDEMONTE DE LOS FARALLONES DE CALI, COLOMBIA
}

\author{
Floristic diversity in a rural landscape of the lower slope \\ in Farallones of Cali, Colombia
}

\author{
Antonella Sardi', Alba Marina Torres ${ }^{2}$ y Germán Corredor ${ }^{3}$
}

Sardi, A., Torres, A. M. y Corredor, G. (2018). Diversidad florística en un paisaje rural del piedemonte de los farallones de Cali, Colombia. Colombia Forestal, 21(2), 142-160

Recepción: 30 de agosto de 2016

\section{Resumen}

El objetivo de esta investigación fue evaluar la composición, estructura y diversidad florística de bosque, matorral y pastizal en un paisaje rural del piedemonte de los Farallones de Cali en el año 2013. Se realizaron muestreos con tres repeticiones en cada elemento del paisaje. En bosque y matorral se muestrearon en total 24 parcelas de $200 \mathrm{~m}^{2}$ para registrar árboles, y 24 subparcelas de $50 \mathrm{~m}^{2}$ para registrar arbustos y plántulas. En pastizales, se muestrearon 36 cuadrantes de $1 \mathrm{~m}^{2}$. En total, se encontraron 140 especies (69 en bosque, 60 en matorral y 53 en pastizal). Las familias con mayor índice de importancia fueron: en bosque Melastomataceae, Myrtaceae y Lacistemataceae; en matorral, Melastomataceae; y en pastizal, Poaceae y Asteraceae. El elemento del paisaje más diverso fue matorral. Se concluye que la comunidad vegetal se encuentra en estados sucesionales tempranos, dominada por plantas pioneras intermedias y ricas en plántulas.

Palabras clave: bosque tropical, composición florística, estructura vegetal, matorral, pastizal.
Aprobación: 22 de marzo de 2018

\begin{abstract}
This study aimed to evaluate composition, structure and plant diversity of forest, scrub and grassland in a rural landscape of lower of the "Farallones of Cali" in 2013. We sampled three replicates of forest and scrub which consisted of a total of 24 plots of $200 \mathrm{~m}^{2}$ to register trees, and 24 subplots of $50 \mathrm{~m}^{2}$ to register shrubs and seedlings. In grassland, 36 quadrants of $1 \mathrm{~m}^{2}$ were sampled. In total, 140 species were recorded (69 in forest, 60 in scrub and 53 in grassland). Families with the highest importance value were Melastomataceae, Myrtaceae and Lacistemataceae in forest, Melastomataceae in scrub and Poaceae and Asteraceae in grassland. In conclusion, the plant community is in early successional stages, dominated by intermediate pioneer species and rich in seedlings plants.
\end{abstract}

Key words: tropical forest, floristic composition, vegetation structure, scrub, grassland.

1 Grupo de Investigación Ecología de Agroecosistemas y Hábitat Naturales (GEAHNA), Universidad del Valle. Cali, Colombia. antonellasardi06@gmail.com. Autor para correspondencia.

2 Grupo de Investigación Ecología y Diversidad Vegetal, Universidad del Valle. Cali, Colombia. alba.torres@correounivalle.edu.co

3 GEAHNA, Universidad del Valle. Cali, Colombia. german.corredor@correounivalle.edu.co 


\section{INTRODUCCIÓN}

La fragmentación del hábitat es considerada una de las principales causas de pérdida de la biodiversidad (Etter y Wyngaarden, 2000; Fahrig, 2003; Rudas et al., 2007; Kattán y Naranjo, 2008), pues en esta la composición, estructura y función original de un ecosistema se han alterado. La pérdida en la conectividad, la creación de bordes en el hábitat o el aislamiento de fragmentos (Fahrig, 2003; Kattán y Naranjo, 2008) provocan dinámicas muy diferentes sobre las poblaciones biológicas que allí se encuentran. Estos factores modifican la composición y abundancia de las especies de un ecosistema e incrementan su vulnerabilidad (Sarmiento et al., 2002; Fahrig, 2003). Se estima que entre el 60 y $75 \%$ de la extensión original de los bosques de los Andes colombianos ha sido transformada a sistemas antropogénicos (Fahrig, 2003). La destrucción de grandes extensiones de bosque da paso a los agroecosistemas característicos de los paisajes rurales, en los que unos parches de bosque de tamaños variables quedan dispersos en una matriz de cultivos, potreros y otros hábitats antropogénicos (Etter, McAlpine, Wilson, Phinn y Possingham, 2006; Kattán y Naranjo, 2008).

El Parque Nacional Natural (PNN) Farallones de Cali es el área protegida más grande del Valle del Cauca, con una extensión de 206770.63 ha y en la que nacen más de 30 ríos que abastecen el suroccidente colombiano. Alberga una gran diversidad florística y abarca varias formaciones vegetales, que van desde el bosque de tierras bajas hasta el páramo (Mayor, et al., 2005). Sin embargo, el área de baja montaña en la zona rural del municipio de Cali no se encuentra protegida y ha sido alterada por diferentes actividades humanas. Este sector es de gran interés, pues los bosques que se encuentran entre los 1000 y $1500 \mathrm{~m}$ de altitud presentan uno de los niveles más altos en concentración de especies por unidad de área (Gentry, 1995; Rangel y Velásquez, 1997).

La vegetación de este lugar ha sido poco estudiada, se sabe que el dosel del bosque puede alcanzar alturas de hasta 35 m y en ellos es común la presencia de palmas y especies de las familias Melastomataceae, Rubiaceae, Lauraceae y Sapotaceae; así como variadas epífitas y helechos arbóreos (Botina y García, 2005; Rodríguez et al., 2006). No obstante, esta zona se encuentra en riesgo debido a la expansión de la frontera urbana, el ecoturismo poco controlado, la minería, incendios forestales y el establecimiento de cultivos y pastos para ganadería. Por ello, todo esto trae como consecuencia un paisaje fragmentado y degradado que amenaza la biodiversidad que alberga.

Los estudios de la composición florística son muy útiles para evaluar la diversidad vegetal en un paisaje fragmentado ya que permiten comparar las comunidades vegetales de los parches en función de su riqueza de especies y evidenciar aspectos de su ecología (Begon, Harper y Townsend, 1999) en relación con otros organismos con los cuales pueden cohabitar. Por otro lado, la estructura de la vegetación responde a varios factores abióticos como la incidencia de la radiación solar, el flujo de la precipitación al interior de la comunidad y la acción del viento (Rangel y Velásquez, 1997). Esta estructura se define por su ordenamiento espacial (Kuchler, 1966), tanto vertical como horizontal (Kuchler, 1966; Rangel y Velásquez, 1997). La estructura vertical se refleja en la estratificación o la altura de los individuos, y la horizontal se manifiesta en la densidad, el área basal y la cobertura vegetal (Rangel y Velásquez, 1997).

La mayoría de los estudios de diversidad en bosques neotropicales se han enfocado en áreas poco intervenidas. Sin embargo, por el interés reciente de entender el efecto de la actividad humana sobre la biodiversidad en paisajes transformados, ha aumentado la cantidad de estudios relacionados con los paisajes rurales (Lozano-Zambrano, 2009). En este contexto, el objetivo de esta investigación fue caracterizar la diversidad florística de un paisaje rural del piedemonte de los Farallones de Cali para entender su composición, estructura y diversidad. Para ello, se evaluaron tres elementos del paisaje, bosques, matorrales y pastizales, predominantes en el área de estudio. 


\section{MATERIALES Y MÉTODOS}

\section{Área de estudio}

La investigación se llevó a cabo en el área rural del municipio de Cali (Colombia), corregimiento de Pance, vereda el Peón ( $\left.3^{\circ} 19^{\prime} 0.7^{\prime \prime} \mathrm{N}-76^{\circ} 34^{\prime} 39^{\prime \prime} \mathrm{O}\right)$, en el área privada Loma Larga, ubicada en el piedemonte del Parque Nacional Natural Farallones. Esta zona se encuentra entre los 1100 y 1250 m de altitud, representa la vegetación secundaria del ecosistema zonobioma alterno hídrico y subxerofítico tropical del Valle del Cauca (Ideam, 2007), con una precipitación y temperatura promedio anual de $1910 \mathrm{~mm}$ y $24.8^{\circ} \mathrm{C}$ respectivamente (Ideam, 2015).

El paisaje está conformado principalmente por parches de bosque secundario (25\%), los cuales se encuentran distribuidos a lo largo de cañadas, matorrales en diferentes estados de sucesión vegetal $(28 \%)$ y pastizales para la ganadería (24\%), considerados los tres elementos o unidades del paisaje a evaluar en este estudio (figura 1). Además, en el paisaje encontramos rastrojos (12\%), que son formaciones densas con alta luminosidad compuesta por plantas herbáceas y arbustos bajos, y en menor proporción helechales $(6 \%)$ y viviendas y vías (5\%) (Corredor, 2016). Dichos parches de bosque son el resultado de la historia de esta región que formó parte de una matriz ganadera y agrícola con cultivos de café, sujeta a incendios forestales, derrumbes, explotación de madera y minería. Los bosques y matorrales se encuentran en proceso de regeneración natural desde hace unos 20 años, bajo un tipo de protección privada por los actuales propietarios.

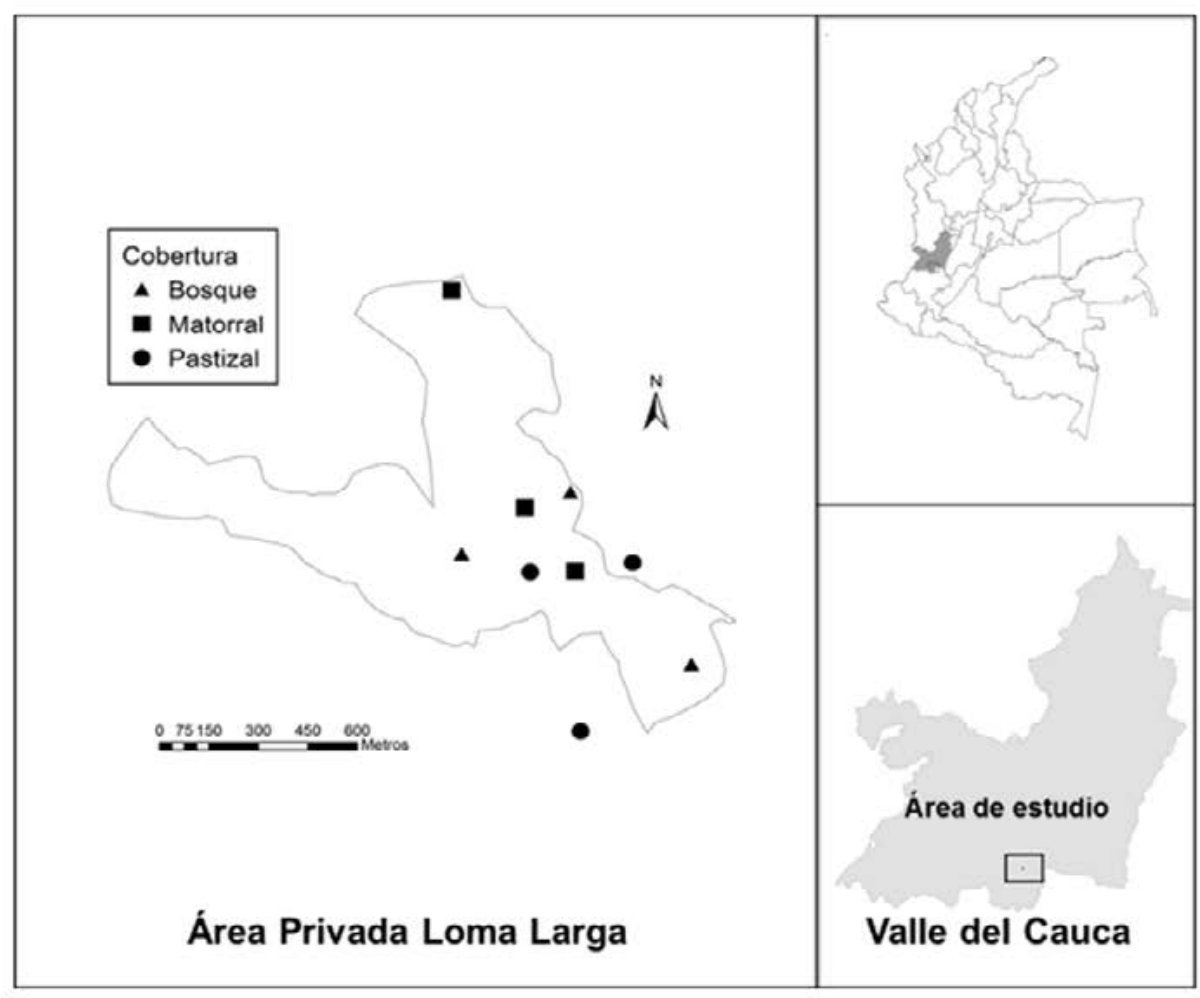

Figura 1. Área de estudio indicando tres repeticiones en cada uno de los elementos del paisaje (bosque, matorral y pastizal). Polígono muestra área de Loma Larga, El Peón, Valle del Cauca, Colombia. 


\section{Muestreo de la vegetación}

Los muestreos fueron realizados en un área de 60 ha entre abril y diciembre de 2013. Para los muestreos en bosque secundario y matorral se caracterizaron los árboles y arbustos empleando el método propuesto por Lozano-Zambrano (2009), el cual consiste en trazar en cada elemento del paisaje cuatro parcelas de $200 \mathrm{~m}^{2}$ (50 $\times 4 \mathrm{~m}$ ), para un total de $800 \mathrm{~m}^{2}$. Se realizaron tres repeticiones por cada cobertura para un total de 24 parcelas (2 elementos del paisaje $\times 4$ parcelas $\times 3$ repeticiones), para un total de $4800 \mathrm{~m}^{2}$.

En cada parcela se hizo el muestreo de individuos con diámetro a la altura del pecho mayor a 5 $\mathrm{cm}$ (i.e. DAP $\geq 5 \mathrm{~cm}$ a $1.3 \mathrm{~m}$ del suelo) que incluyó árboles, arbustos y lianas. Además, se realizaron al interior de cada parcela muestreos de vegetación en subparcelas de $50 \mathrm{~m}^{2}(50 \times 1 \mathrm{~m})$, para un total de 24 subparcelas $\left(1200 \mathrm{~m}^{2}\right)$. En estas subparcelas se registraron los individuos leñosos y con diámetro entre 0.5 y $2.5 \mathrm{~cm}$, medido a $50 \mathrm{~cm}$ del suelo, e incluye arbustos y plántulas. Estas dos categorías diametrales muestran el componente arbóreo (i.e. $\mathrm{DAP} \geq 5 \mathrm{~cm}$ ) y la regeneración de la comunidad vegetal (i.e. DAP 0.5 a $2.5 \mathrm{~cm}$ ). Para cada individuo se registró el nombre común, el hábito de crecimiento, la circunferencia a la altura del pecho (CAP) y características como el tipo de corteza, aromas, entre otros para facilitar su identificación taxonómica.

En cada pastizal se hicieron dos transectos de $50 \mathrm{~m}$ de longitud cada uno, distanciados entre sí por $50 \mathrm{~m}$. En cada transecto se ubicaron seis subparcelas de $1 \mathrm{~m}^{2}$ para un total de $12 \mathrm{~m}^{2}$ por cada pastizal. Se muestrearon tres pastizales, para un total de 36 parcelas $\left(36 \mathrm{~m}^{2}\right)$.

De cada especie registrada, se tomaron fotografías, se colectaron dos ejemplares, que fueron depositados en el herbario de la Universidad del Valle (CUVC), bajo la serie AS. Los especímenes fueron identificados en su mayoría hasta la categoría taxonómica específica, por comparación en colecciones de herbario, mediante literatura especializada o con la ayuda de especialistas.

\section{Análisis de datos}

Para cada sitio de muestreo se determinó la abundancia y la riqueza total por especies y por familias de plantas. Para los individuos con DAP $\geq 5 \mathrm{~cm}$ de bosque y matorral se calculó el índice de valor de importancia (IVI), así como la sumatoria de la densidad relativa (DR), cobertura relativa $(\mathrm{CR})$ y frecuencia relativa (FR). También se calculó el índice de valor de importancia por familias (IVIF) para estimar las familias dominantes (Rangel y Velásquez, 1997). Adicionalmente, se graficó una distribución de tallos por clase diamétricas con el fin de determinar el estado sucesional.

Para los individuos con DAP entre 0.5 y $2.5 \mathrm{~cm}$ y todos los individuos registrados en los pastizales, se estimó la densidad y la frecuencia relativa (Rangel y Velásquez, 1997).

La diversidad se midió como el número de especies efectivas mediante el cálculo de la diversidad verdadera (qD) propuesta por Jost (2006), con la fórmula:

$$
{ }^{q} D=\left(\sum_{i=1}^{S} P_{i}^{q}\right)^{1 /(-q)}
$$

Donde, pi es la abundancia relativa (i.e. abundancia proporcional) de la i-ésima especie, $\mathrm{S}$ es el número de especies (i.e. riqueza) y q es el orden de la diversidad y define la sensibilidad del índice a las abundancias relativas de las especies (Jost, 2007). El valor del parámetro q determina la influencia de la abundancia de las especies en la medida de la diversidad y puede tomar cualquier valor de 0 a infinito. Para este estudio se tomó un valor de $q=1\left({ }^{1} \mathrm{D}\right.$, exponencial de la entropía de Shannon, que da igual peso a todas las especies) (Jost 2006).

Finalmente, se hizo un análisis no métrico multidimensional (NMDS) para determinar la similitud entre parcelas usando los valores de abundancia de las especies; se usó la distancia Bray curtis, dos dimensiones y stress $=0.1205192$, en el programa estadístico R, librería fun (R Core Team, 2013). 


\section{RESULTADOS}

\section{Diversidad y composición florística}

Los tres elementos del paisaje del área de Loma Larga se caracterizan por:

Bosques secundarios: dispuestos principalmente a lo largo de cañadas, con un dosel cerrado, predominio de árboles con alturas que oscilan entre 15 y $25 \mathrm{~m}$, tres estratos verticales, sotobosque medianamente denso, rico en plántulas en crecimiento y suelo recubierto $100 \%$ por hojarasca. La diversidad en el bosque tuvo valores intermedios, comparados con las otras dos unidades del paisaje matorrales y pastizales $\left(\mathrm{B}_{1}{ }^{1} \mathrm{D}=9.14 ; \mathrm{B}_{2}{ }^{1} \mathrm{D}=15.53 ; \mathrm{B}_{3}{ }^{1} \mathrm{D}=14.73\right)$.

Matorrales: presenta árboles dispersos y distribuidos en parches, con variación en la cobertura arbórea, desde matorrales abiertos hasta matorrales semi-cerrados. Los arbustos y árboles tienen alturas entre 5 y $15 \mathrm{~m}$, con vegetación densa al interior, suelo cubierto de herbáceas en un $80 \%$ y con algunas plántulas de árboles. La diversidad en el matorral tuvo los valores más altos entre los tres elementos del paisaje $\left(M_{1}{ }^{1} D=20.22 ; M_{2}{ }^{1} D=\right.$ 20.69; $M_{3}{ }^{1} \mathrm{D}=18.1$ ).

Pastizales: forman parte de potreros y senderos ecoturísticos, con predominio de poáceas. Los potreros tienen ganadería poco intensiva y control de herbáceas con guadaña o con machete. La diversidad en el pastizal fue la menor entre los tres elementos del paisaje $\left(\mathrm{P}_{1}{ }^{1} \mathrm{D}=8.04 ; \mathrm{P}_{2}{ }^{1} \mathrm{D}=4.17\right.$; $\mathrm{P}_{3}{ }^{1} \mathrm{D}=2.84$ ).

En los tres elementos del paisaje se encontraron en total 140 especies de plantas, pertenecientes a 43 familias (anexo 1). En el elemento del paisaje bosque, se registraron 69 especies, 55 géneros y 30 familias, representadas por 32 especies 27 géneros y 20 familias con DAP $\geq 5 \mathrm{~cm}$ y 61 especies, 49 géneros y 27 familias con DAP entre $0.5 \mathrm{~cm}$ y $2.5 \mathrm{~cm}$ (tabla 1).

En matorral se registraron 60 especies, 42 géneros y 24 familias; donde 23 especies, 19 géneros y 12 familias presentaron DAP $\geq 5 \mathrm{~cm}$ y 55 especies, 36 géneros y 23 familias registraron DAP entre 0.5 $\mathrm{cm}$ y $2.5 \mathrm{~cm}$. En pastizal se registraron en total 53 especies de hierbas y plántulas, 43 géneros y 18 familias (tabla1).

En bosque, dentro de la categoría cuyos individuos tuvieron DAP mayor o igual a $5 \mathrm{~cm}$, las familias mejor representadas fueron Lauraceae, con tres géneros y cinco especies, y Melastomataceae, con dos géneros y tres especies. Las familias más abundantes fueron Melastomataceae, Myrtaceae, Lacistemataceae, Erythroxylaceae y Lauraceae. Las especies dominantes fueron Lacistema aggregatum (P.J. Bergius) Rusby, Eugenia cf. egensis DC., Henriettea seemannii (Naudin) L.O. Williams y Erythroxylum citrifolium A. St.-Hil. Dentro de la categoría cuyos individuos tuvieron DAP entre 0.5 y $2.5 \mathrm{~cm}$, las familias con mayor número de especies fueron Melastomataceae, Rubiaceae y Piperaceae, cada una con seis especies. En abundancia, las familias que se destacaron fueron Erythroxylaceae y Myrtaceae. Las especies más abundantes fueron E. cf. egensis y E. citrifolium.

En matorral, dentro de la categoría de individuos con DAP mayor o igual a $5 \mathrm{~cm}$, la familia con mayor riqueza de especies y a su vez la más abundante fue Melastomataceae, representada

Tabla 1. Abundancia y riqueza de plantas dentro de dos categorías de DAP, en tres elementos del paisaje de Loma Larga, El Peón, Valle del Cauca, Colombia, entre abril y diciembre de 2013.

\begin{tabular}{|c|c|c|c|c|c|c|}
\hline \multirow{3}{*}{$\begin{array}{c}\text { Elemento del } \\
\text { paisaje }\end{array}$} & \multicolumn{3}{|c|}{$0.5 \mathrm{~cm} \leq \mathrm{DAP} \leq 2.5 \mathrm{~cm}$} & \multicolumn{3}{|c|}{$\mathrm{DAP} \geq 5 \mathrm{~cm}$} \\
\hline & \multirow{2}{*}{$\begin{array}{c}\text { Abundancia } \\
\text { (n. }{ }^{\circ} \text { individuos) }\end{array}$} & \multicolumn{2}{|c|}{ Riqueza } & \multirow{2}{*}{$\begin{array}{c}\text { Abundancia } \\
\text { (n. }^{\circ} \text { individuos) }\end{array}$} & \multicolumn{2}{|c|}{ Riqueza } \\
\hline & & ( $n .^{\circ}$ especies) & (n. ${ }^{\circ}$ familias $)$ & & (n. ${ }^{\circ}$ especies) & (n..$^{\circ}$ familias) \\
\hline Bosque & 1168 & 61 & 27 & 251 & 32 & 20 \\
\hline Matorral & 889 & 55 & 20 & 142 & 23 & 12 \\
\hline Pastizal & 0 & 56 & 17 & 0 & 0 & 0 \\
\hline
\end{tabular}


por dos géneros y seis especies. Las especies más abundantes en este caso fueron Ladenbergia oblongifolia (Humb. ex Mutis) L. Andersson, Miconia minutiflora (Bonpl.) DC. y Miconia rubiginosa (Bonpl.) DC. Para la categoría con individuos con DAP entre 0.5 y $2.5 \mathrm{~cm}$, las familias con mayor riqueza de especies fueron Asteraceae (10) y Melastomataceae (9). La familia más abundante fue Melastomataceae y las especies más abundantes fueron M. minutiflora, E. cf. egensis, y Miconia stenostachya DC.

En pastizal, las familias con mayor riqueza y densidad fueron Poaceae (10 especies) y Asteraceae (9). Las especies más abundantes fueron Urochloa decumbens (Stapf) R.D. Webster, Eleutheranthera sp. y Homolepis aturensis (Kunth) Chase.

Por otra parte, el análisis NMDS permitió observar la separación de tres grandes grupos, que representan los tres elementos del paisaje bosque, matorral y pastizal. Estos resultados confirman la categorización subjetiva que, a priori, se había hecho de los elementos del paisaje. Las parcelas de bosque fueron más homogéneas entre sí, mientras que matorral y pastizal tuvieron más diferencias entre parcelas (figura 2).

El pastizal se diferenció completamente de bosques y matorrales. Las diferencias entre parcelas se dieron debido a los diferentes usos que tiene cada pastizal. Por un lado, el pastizal 3 tuvo una gran intensidad ganadera, como se pudo evidenciar por la dominancia del pasto para ganadería $U$. decumbens. Mientras que los pastizales 1 y 2 , con presencia ocasional o ausencia de ganadería, presentaron una mayor riqueza de arvenses de las familias Poaceae, Asteraceae y Fabaceae, con especies propias de pastizal como Hyparrhenia rufa (Nees) Stapf, Pseudelephantopus spiralis (Less.) Cronquist y Mimosa púdica L.

Dentro de los materiales había tres subgrupos: matorral temprano, matorral y matorral avanzado.

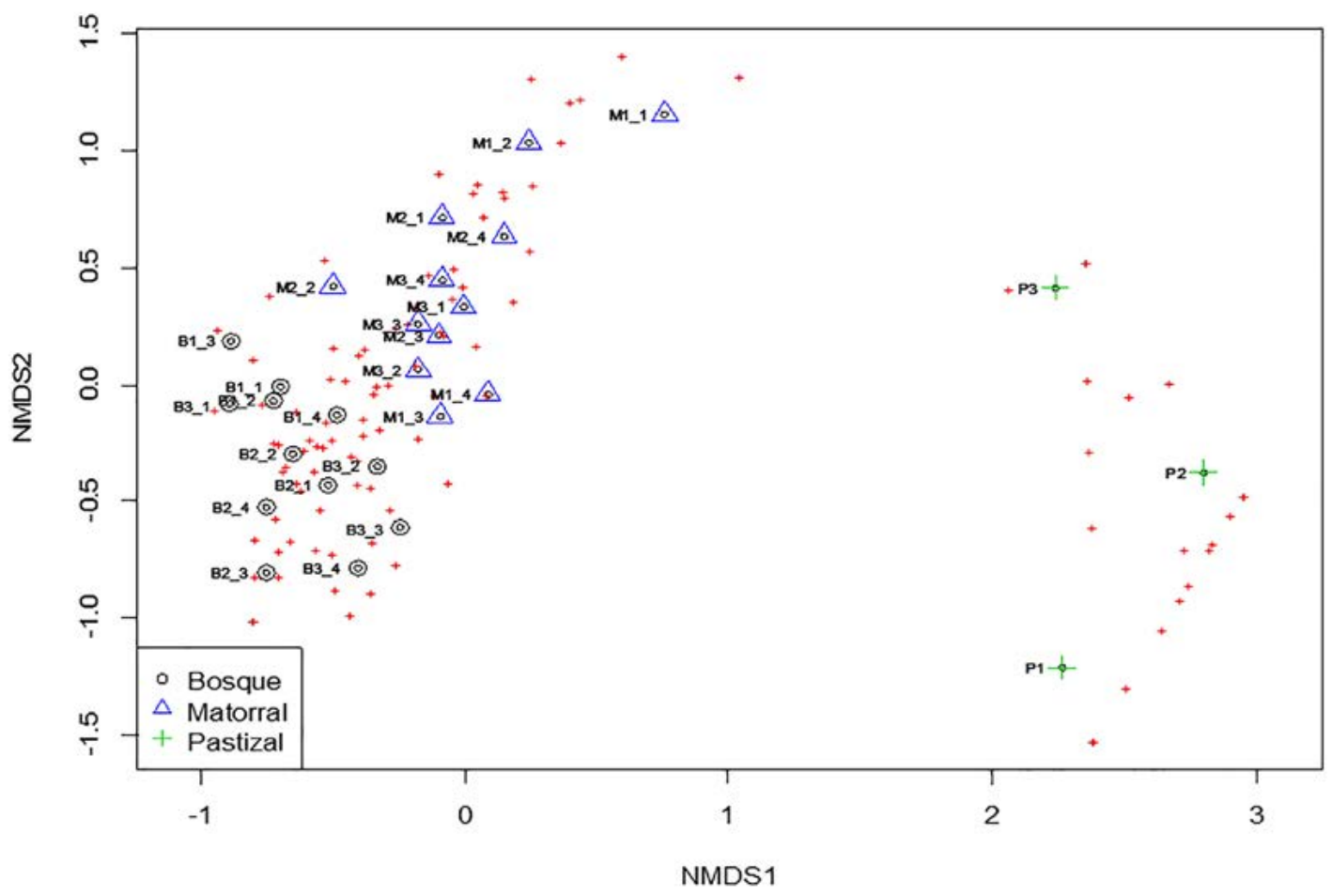

Figura 2. Análisis no métrico multidimensional (NMDS) basado en la abundancia de las especies de las subparcelas para los tres elementos del paisaje, B: bosque; M: matorral; P: pastizal, en Loma Larga, El Peón, Valle del Cauca, Colombia, entre abril y diciembre de 2013. 
El primero se caracterizó por presentar arbustos aislados del género Miconia, como M. minutiflora y $M$. rubiginosa, además del dominio de herbáceas de las familias Asteraceae (e.g. Chromolaena tacotana (Klatt) R.M. King \& H. Rob.) y Melastomataceae (e.g. M. stenostachya, Clidemia ciliata D. Don) y la colonización de plántulas del género Miconia. El matorral estuvo conformado por arbustos con géneros como Miconia, Clusia y árboles dispersos como L. oblongifolia y Persea caerulea (Ruiz \& Pav.) Mez. El matorral avanzado se puede considerar como una transición entre bosque y matorral, en el cual hay árboles de mayor porte y un sotobosque tapizado por herbáceas de las familias Asteraceae y Poaceae, además de la colonización de plántulas de especies propias de bosque como E. citrofolium. Entre las especies características de esta transición se encuentran Cinnamomum triplinerve (Ruiz \& Pav.) Kosterm, Schefflera morototoni (Aubl.) Maguire, Steyerm. \& Frodin, Ocotea aurantiodora (Ruiz \& Pav.) Mez y H. seemanii.

Los bosques fueron más homogéneos entre sí y se caracterizaron por el predominio de árboles como Calliandra pittieri Standl., Miconia matthaei Naudin, E. citrifolium y Allophylus racemosus Sw. Mientras que el sotobosque estuvo constituido por las plántulas de dichos árboles, y por hierbas y arbustos de los géneros Psychotria y Piper.

\section{Estructura de la vegetación}

Teniendo en cuenta solo los individuos con DAP superior a $5 \mathrm{~cm}$, se encontró que $H$. seemannii, $S$. morototoni y $O$. aurantiodora estuvieron entre las especies con mayor IVI, tanto en bosque como en matorral.

En bosque, las especies con mayor distribución espacial, es decir, mayor frecuencia relativa (FR), fueron $E$. cf. egensis (FR = 11.0), H. seemannii (FR $=8.4)$ y $L$. aggregatum $(F R=8.4)$. Las especies con mayor cobertura fueron $H$. seemannii $(\mathrm{CR}=$ 24.3), Cupania americana L. (12.7) y C. pittieri $(C R=10.3)$. Asimismo, las especies con mayor índice de valor de importancia (IVI) fueron $H$. seemannii $(\mathrm{IVI}=46.2)$, L. aggregatum $(\mathrm{IVI}=29.9)$ y E. cf. egensis $(\mathrm{IVI}=29.7)$. Por otro lado, las familias con mayor índice de valor de importancia (IVIF) fueron Melastomataceae (IVIF = 60.6), Myrtaceae (IVIF = 34.5) y Lacistemataceae (IVIF = 31.8) (tabla 2).

En matorral, las especies con mayor distribución espacial fueron $H$. seemannii $(F R=12.0), M$. minutiflora ( $\mathrm{FR}=10.0)$, L. oblongifolia $(\mathrm{FR}=8.8)$ y S. morototoni $(\mathrm{FR}=8.8)$. Las especies con mayor cobertura fueron $L$. oblongifolia $(\mathrm{CR}=23.0)$, Pinus patula Schltdl. \& Cham. (CR =13.4), y H. seemannii $(\mathrm{CR}=10.7)$. Las especies con mayor IVI fueron $L$. oblongifolia $(\mathrm{IVI}=46.6), \mathrm{H}$. seemannii $(\mathrm{IVI}=32.4)$ y M. minutiflora (IVIF $=28.4$ ) (tabla 2). Las familias más importantes fueron Melastomataceae $(I V I F=85.9)$, Rubiaceae $(I V I F=49.6)$ y Araliaceae $(\mathrm{IVIF}=31.3)$.

Para los individuos con DAP entre $0.5 \mathrm{~cm}$ y $2.5 \mathrm{~cm}$, las especies E. cf. egensis y E. citrifolium, se destacaron por presentar mayor IVI tanto en bosque como en matorral. Siendo $E$. cf. egensis la especie con mayor IVI en los dos elementos del paisaje.

En bosque, las especies con mayores frecuencias fueron $E$. cf. egensis ( $F R=5.9)$, E. citrifolium $(\mathrm{FR}=5.4)$, L. aggregatum $(\mathrm{FR}=5.4)$ y Psychotria deflexa D.C. (FR = 5.4). La especie con mayor cobertura fue $E$. $c f$. egensis $(C R=32.1)$ y la familia más importante fue Myrtaceae (IVIF = 89.8).

En matorral, las especies más frecuentes fueron M. minutiflora ( $\mathrm{FR}=5.3)$, E. citrifolium ( $\mathrm{FR}=4.9), E$. cf. egensis (FR $=4.9$ ) y Ocotea aurantiodora (Ruiz \& Pav.) Mez (FR = 4.9). La especie con mayor cobertura fue $M$. rubiginosa $(C R=18.1)$ y la familia más importante fue Melastomataceae (IVIF = 77.2) (tabla 3).

En pastizal, las especies más frecuentes fueron $U$. decumbens $(\mathrm{FR}=9.8)$ y Eleutheranthera sp. $(F R=8.8)$, predominando $U$. decumbens con una densidad relativa de 36.5 (tabla 3).

La distribución de los tallos de los árboles de bosque y matorral por clases diamétricas mostró una curva en forma de J invertida (figura 3). 
Tabla 2. Lista de las especies con mayor IVI (DAP $\geq 5 \mathrm{~cm}$ ) en los elementos del paisaje bosque y matorral de Loma Larga, El Peón, Valle del Cauca, Colombia, entre abril y diciembre de 2013. DR: densidad relativa; FR: frecuencia relativa; CR: cobertura relativa; IVI: índice de valor de importancia por especie; IVIF: índice de valor de importancia por familia.

\begin{tabular}{|c|c|c|c|c|c|c|c|}
\hline & Familia & Especie & DR & FR & CR & IVI & IVIF \\
\hline \multirow{10}{*}{ Bosque } & Melastomataceae & Henriettea seemannii & 13.5 & 8.4 & 24.3 & 46.2 & 60.6 \\
\hline & Lacistemataceae & Lacistema aggregatum & 15.1 & 8.4 & 6.4 & 29.9 & 31.8 \\
\hline & Myrtaceae & Eugenia cf. egensis & 15.1 & 11.0 & 3.3 & 29.7 & 34.5 \\
\hline & Sapindaceae & Cupania americana & 3.6 & 4.7 & 12.7 & 21.0 & 25.4 \\
\hline & Fabaceae & Calliandra pittieri & 4.0 & 5.6 & 10.3 & 19.9 & 21.1 \\
\hline & Erythroxylaceae & Erythroxylum citrifolium & 10.0 & 5.6 & 4.0 & 19.6 & 20.8 \\
\hline & Lauraceae & Ocotea aurantiodora & 6.4 & 5.6 & 5.8 & 17.8 & 28.6 \\
\hline & Melastomataceae & Miconia matthaei & 7.6 & 6.5 & 2.0 & 16.1 & 60.6 \\
\hline & Araliaceae & Schefflera morototoni & 4.0 & 4.7 & 6.7 & 15.4 & 22.2 \\
\hline & Clusiaceae & Clusia palmicida & 1.2 & 2.8 & 7.3 & 11.3 & 12.4 \\
\hline \multirow{10}{*}{ Matorral } & Rubiaceae & Ladenbergia oblongifolia & 14.8 & 8.8 & 23.0 & 46.6 & 49.6 \\
\hline & Melastomataceae & Henriettea seemannii & 9.9 & 12.0 & 10.7 & 32.4 & 85.9 \\
\hline & Melastomataceae & Miconia minutiflora & 12.7 & 10.0 & 5.4 & 28.4 & 85.9 \\
\hline & Melastomataceae & Miconia rubiginosa & 12.7 & 7.4 & 5.5 & 25.5 & 85.9 \\
\hline & Araliaceae & Schefflera morototoni & 5.6 & 8.8 & 9.8 & 24.2 & 31.3 \\
\hline & Primulaceae & Myrsine pellucida & 8.5 & 5.9 & 6.0 & 20.3 & 22.3 \\
\hline & Pinaceae & Pinus patula & 0.7 & 1.5 & 13.4 & 15.6 & 16.1 \\
\hline & Myrtaceae & Myrcia popayanensis & 4.9 & 4.4 & 4.0 & 13.3 & 31.0 \\
\hline & Lauraceae & Persea caerulea & 4.2 & 4.4 & 2.8 & 11.5 & 27.6 \\
\hline & Lauraceae & Ocotea aurantiodora & 2.8 & 5.9 & 1.4 & 10.1 & 27.6 \\
\hline
\end{tabular}

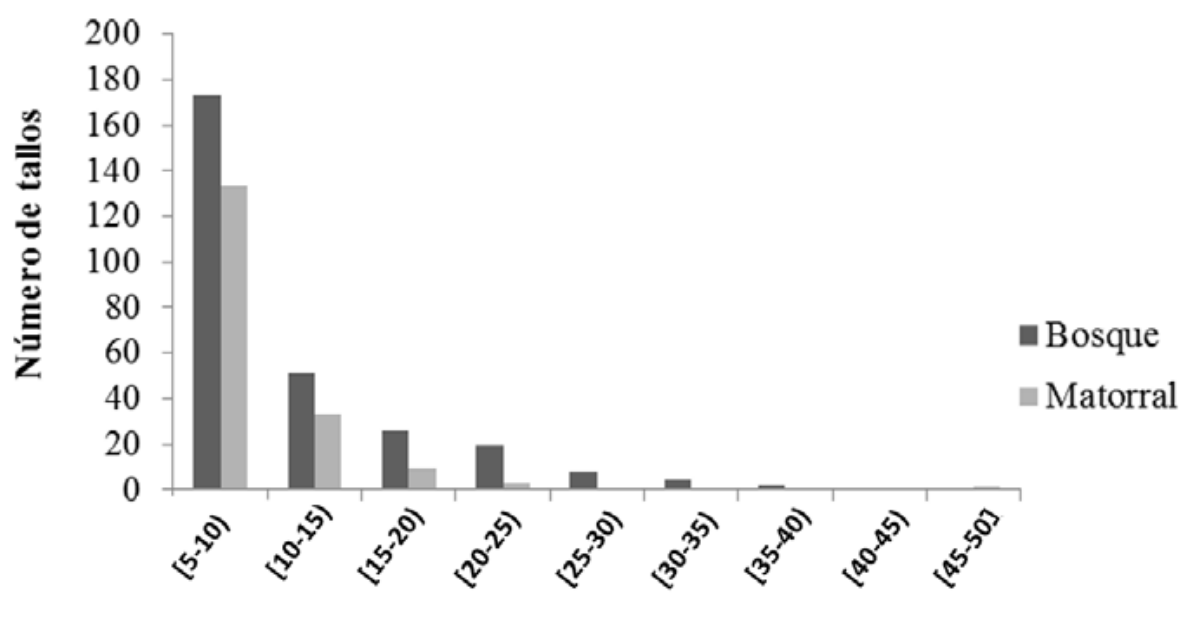

\section{DAP (cm)}

Figura 3. Distribución de tallos de los árboles de bosque y matorral por clases diamétricas en Loma larga, El Peón, Valle del Cauca, Colombia, entre abril y diciembre de 2013. 
Tabla 3. Lista de las especies con mayor IVI $(0.5 \mathrm{~cm} \leq \mathrm{DAP} \leq 2.5 \mathrm{~cm})$ en bosque y matorral y mayor abundancia en pastizal de Loma Larga, El Peón, Valle del Cauca, Colombia, entre abril y diciembre de 2013. DR: densidad relativa; FR: frecuencia relativa; CR: cobertura relativa; IVI: índice de valor de importancia por especie; IVIF: índice de valor de importancia por familia.

\begin{tabular}{|c|c|c|c|c|c|c|c|}
\hline & Familia & Especie & DR & FR & CR & IVI & IVIF \\
\hline \multirow{10}{*}{ Bosque } & Myrtaceae & Eugenia cf. egensis & 32.7 & 5.9 & 32.1 & 70.7 & 89.8 \\
\hline & Erythroxylaceae & Erythroxylum citrifolium & 12.8 & 5.4 & 11.8 & 30.0 & 32.4 \\
\hline & Lacistemataceae & Lacistema aggregatum & 3.9 & 5.4 & 9.1 & 18.5 & 20.8 \\
\hline & Myrtaceae & Syzygium jambos & 8.1 & 2.9 & 5.6 & 16.6 & 89.8 \\
\hline & Rubiaceae & Psychotria deflexa & 4.9 & 5.4 & 2.7 & 12.9 & 23.4 \\
\hline & Poaceae & Olyra latifolia & 2.0 & 2.0 & 2.5 & 10.6 & 8.67 \\
\hline & Arecaceae & Prestoea acuminata & 3.0 & 3.4 & 3.4 & 9.9 & 14.5 \\
\hline & Primulaceae & Parathesis candolleana & 6.6 & 2.5 & 6.6 & 9.4 & 18.1 \\
\hline & Lauraceae & Ocotea aurantiodora & 1.8 & 3.9 & 2.3 & 8.3 & 12.8 \\
\hline & Rubiaceae & Coffea arabica & 2.2 & 2.0 & 3.3 & 7.5 & 23.4 \\
\hline \multirow{10}{*}{ Matorral } & Myrtaceae & Eugenia cf. egensis & 8.9 & 4.9 & 10.5 & 24.3 & 41.7 \\
\hline & Melastomataceae & Miconia rubiginosa & 1.6 & 1.9 & 18.1 & 21.6 & 77.2 \\
\hline & Erythroxylaceae & Erythroxylum citrifolium & 6.3 & 4.9 & 7.7 & 18.9 & 22.3 \\
\hline & Melastomataceae & Miconia minutiflora & 10.2 & 5.3 & 1.1 & 16.6 & 77.2 \\
\hline & Melastomataceae & Clidemia ciliata & 6.7 & 3.9 & 3.3 & 13.9 & 77.2 \\
\hline & Clusiaceae & Clusia minor & 4.0 & 3.4 & 5.2 & 12.6 & 15.0 \\
\hline & Poaceae & Guadua angustifolia & 6.1 & 1.0 & 5.4 & 12.4 & 22.8 \\
\hline & Lauraceae & Ocotea aurantiodora & 5.6 & 4.9 & 1.9 & 12.4 & 19.8 \\
\hline & Melastomataceae & Miconia stenostachya & 7.9 & 3.9 & 0.4 & 12.1 & 77.2 \\
\hline & Myrtaceae & Myrcia popayanensis & 4.7 & 2.4 & 4.5 & 11.6 & 41.7 \\
\hline \multirow{10}{*}{ Pastizal } & Poaceae & Urochloa decumbens & 36.5 & 9.8 & - & - & - \\
\hline & Asteraceae & Eleutheranthera sp. & 13.5 & 8.8 & - & - & - \\
\hline & Poaceae & Homolepis aturensis & 10.7 & 2.9 & - & - & - \\
\hline & Poaceae & Steinchisma laxum & 5.2 & 3.4 & - & - & - \\
\hline & Asteraceae & Emilia fosbergii & 3.8 & 4.4 & - & - & - \\
\hline & Poaceae & Oplismenus sp. & 3.3 & 2.5 & - & - & - \\
\hline & Asteraceae & Pseudelephantopus spiralis & 2.6 & 3.4 & - & - & - \\
\hline & Fabaceae & Desmodium sp. 2 & 2.2 & 3.9 & - & - & - \\
\hline & Poaceae & Digitaria sp. & 1.91 & 2.0 & - & - & - \\
\hline & Poaceae & Hyparrhenia rufa & 1.70 & 2.9 & - & - & - \\
\hline
\end{tabular}

El $74.3 \%$ de los individuos en bosque y el $61.1 \%$ en matorral se ubicaron en la clase diamétrica de 5-10 cm. Se registraron más individuos en la clase con diámetro menor y disminuyeron significativamente a medida que aumenta el diámetro. En pastizales no se registraron árboles.

\section{DISCUSIÓN}

\section{Diversidad y composición florística}

La riqueza y diversidad vegetal resultado de la recuperación de bosque y matorral muestran la resiliencia de este paisaje, con mayoría de especies 
pioneras, aunque no evidencia elementos del bosque original.

El elemento del paisaje matorral mostró los valores más altos de diversidad y comparte $50 \%$ de las especies con bosque y $17 \%$ con pastizal. En contraste, el bosque presentó valores más bajos de diversidad que el matorral, pues está dominado por pocas especies, a pesar de que tuvo mayor riqueza, lo cual es frecuente en bosques tropicales en los que procesos de dispersión de semillas son limitados y, por lo tanto, se generan distribuciones agrupadas de individuos de una misma especie (Hubbell, 1979). Los valores más bajos de diversidad los presentaron los pastizales por ser más homogéneos y estar dominados por especies de Poaceae.

Los bosques presentaron homogeneidad entre subparcelas. Los bosques 1 y 3 pertenecen a la microcuenca La Quesada y el bosque 2 se localiza en la microcuenca La Mina. En general, la composición de las especies de las subparcelas de cada bosque es similar debido a la proximidad de los fragmentos y al estado sucesional en que se encuentran.

Por el contrario, los matorrales, aunque forman un grupo separado de bosques y pastizales, mostraron mayor heterogeneidad entre ellos, incluso entre las subparcelas de cada matorral. Esto responde a la variación microclimática y a la mayor tasa de colonización de este elemento del paisaje.

La variación entre estos elementos del paisaje puede estar determinada por la variación de factores abióticos (i.e. tipo de suelo y pendiente del suelo) y bióticos (i.e. dispersión y depredación), como a la historia de uso de cada unidad del paisaje, con diferentes estados sucesionales originadas por distintas alteraciones antrópicas.

La riqueza de especies en bosque, con DAP > $2.5 \mathrm{~cm}$, reportada para este estudio (i.e. 69) es relativamente baja con relación a otros bosques premontanos de Colombia, en Antioquia, Magdalena y Valle del Cauca, en los que se encontraron entre 56 y 150 especies (Gentry, 1995; Cantillo y Fajardo, 2004; Robles, 2006; Ariza, Toro y Lores, 2009). Sin embargo, la riqueza es similar o mayor que en los estudios en bosques secos del Valle del Cauca (Torres et al., 2012; Londoño y Torres, 2015).

La composición florística del bosque en Loma Larga tiene una influencia muy grande de especies del bosque seco, ya que más del $62 \%$ de las especies registradas se han reportado en este tipo de bosques (Vargas, 2012). Sin embargo, familias tan predominantes del bosque seco no son muy representativas en Loma larga como las Fabáceas, Rutáceas y Moráceas (Torres et al., 2012; Vargas, 2012). Mientras que familias de bosques premontanos de los Andes como Myrtaceae y Melastomataceae (Cantillo y Fajardo, 2004; Robles, 2006; Ariza et al., 2009; Cháves y Rodríguez, 2012; Trujillo y Henao, 2018), fueron dominantes en el área de estudio. Las especies arbóreas que predominaron en el bosque H. seemannii, L. aggregatum y $E$. cf. egensis son propias de bosques húmedos de zonas bajas o montañosas (Giraldo-Castaño, 2000; Reina, Medina, Ávila, Ángel y Cortéz, 2010; Cháves y Rodríguez, 2012; Vásquez-Vélez, 2014). Este patrón confirma que esta zona representa la transición entre el bosque seco y el bosque premontano (Botina y García, 2005).

En bosque, para los individuos con DAP inferior a $2.5 \mathrm{~cm}$, las familias con mayor riqueza de especies fueron Piperaceae, Rubiaceae y Melastomataceae. Dichas familias presentan generalmente alta riqueza en el sotobosque de los bosques de los Andes (Laska, 1997; Botina y García, 2005; Villarreal et al., 2006). La riqueza de plántulas y arbustos (i.e. DAP $<2.5 \mathrm{~cm}$ ) fue superior a la riqueza de árboles (i.e. DAP $>2.5 \mathrm{~cm}$ ), evidenciando que estos son bosques jóvenes. En general, el número de especies de plantas de sotobosque aumenta en los primeros estados sucesionales y va disminuyendo con la edad; mientras que la diversidad de árboles, por el contrario, aumenta con la edad (Gentry, 1982; Laska, 1997). Las plantas de sotobosque tienen patrones de diversidad diferentes a los que presentan los árboles, ya que difieren en las variables de respuesta a factores abióticos como diferentes niveles de luz, nutrientes y disponibilidad de agua, viento y temperatura (Denslow, Schultz, 
Vitousek y Strain, 1990). El sotobosque se caracterizó por estar poblado por una gran cantidad de plántulas de diferentes especies arbóreas (1168 individuos), lo cual muestra el alto reclutamiento que ocurre en estos bosques jóvenes.

En contraste, en matorral hubo un menor número de individuos entre $0.5 \mathrm{~cm}$ y $2.5 \mathrm{~cm}$ de DAP que lo encontrado en bosque. Este elemento del paisaje está cubierto por pastos secos y helechos que cubren el suelo e inhiben el crecimiento de otras plantas. En matorral es común el reemplazo de pastos por arbustos ampliamente espaciados que pueden amortiguar las drásticas condiciones ambientales y beneficiar el establecimiento y desarrollo de plántulas (Browning, Laliberte y Rango, 2011). Aunque en Loma Larga el matorral está dominado por arbustos tolerantes a la luz, la mayoría de la familia Melastomataceae, una buena proporción de las plántulas que empiezan a colonizar el suelo son especies que dominan el bosque (como E. cf. egensis y E. citrifolium). De hecho, especies de borde de bosque de los géneros Eugenia y Miconia, son registradas como colonizadoras de pastizales abandonados en Puerto Rico (Cubiña y Aide, 2001). Estos arbustos colonizadores son de gran importancia en la regeneración natural de la vegetación, pues traen consigo la acumulación de materia orgánica, la cual aumenta los nutrientes del suelo y aumenta las partículas transportadas por el agua; esto trae como consecuencia la formación de sistemas de raíces, el incremento de los sitios de germinación y el establecimiento de plántulas (Montaña, 1992).

Por otra parte, la riqueza de los pastizales es relativamente alta (i.e. 56), lo cual indica que tienen baja presión de intervención y pastoreo. Estos están dominados por las familias Poaceae y Asteraceae. Las especies de Asteraceae son clave en los procesos de colonización de áreas abiertas, en la creación de hábitat en estados iniciales de sucesión y en el mejoramiento de las condiciones para el establecimiento de especies con altos requerimientos de luminosidad (Lozano-Zambrano, 2009).

\section{Estructura de la vegetación}

A nivel estructural, en los bosques y los matorrales pocas especies fueron muy abundantes (i.e. mayor IVI) y muchas especies tuvieron pocos individuos (i.e. menos IVI). La especie H. seemannii, fue la de mayor IVI dentro del bosque por su alta frecuencia, densidad y su gran porte, mientras que L. aggregatum y $E$. cf. egensis, las especies que le siguen en orden de importancia, determinaron su dominancia por su alta densidad y frecuencia. En los matorrales, L. oblongifolia fue la especie más importante por su gran cobertura y su densidad, aunque no fue tan frecuente en las parcelas. Mientras que el valor de importancia de $H$. seemannii se debe a la frecuencia y cobertura y la de $M$. minutiflora a la frecuencia y densidad.

La distribución de clases diamétricas de tallos en forma de $\mathrm{J}$ invertida tanto en bosque como en matorral, con DAP entre 5 y $10 \mathrm{~cm}$ estuvieron sobre-representadas, es decir, exhibieron un alto número de individuos, lo que es característica de estados sucesionales tempranos (Torres et al., 2012).

\section{Ecología de las especies}

El bosque estuvo dominado por especies pioneras intermedias, propias de bosques secundarios tardíos, que toleran la sombra, pero necesitan luz para alcanzar mayores tallas, tales como especies típicas de claro de bosques (Hubbell, Foster y O’Brien, 1999). Por ejemplo, H. seemannii fue la especie más importante del estudio y especies con alto IVI como L. aggregatum, E. cf. egensis, C. americana, C. pittieri y E. citrifolium, han sido reportadas como pioneras intermedias en bosques del Huila (Vargas, 2015). Especies como estas son muy buenas competidoras, eficientes en la acumulación de biomasa, producción de hojarasca, creación de hábitat, producen grandes cantidades de recursos para la fauna y son tolerantes a condiciones extremas de clima (Lozano-Zambrano, 2009; Vargas, 2015). 
En contraste, en pastizal, las especies pioneras o heliófitas (Swaine y Whitmore, 1988) como las especies herbáceas de Poaceae y Asteraceae presentaron la mayor densidad. Sin embargo, en algunos lugares donde hay un poco de sombra ocurren especies como C. americana y Croton gossypiifolius Vahl.

Por otra parte, las plantas cultivadas o introducidas están representadas por pocas especies en los tres elementos del paisaje. El matorral fue el que presentó un mayor número de especies introducidas, encontrándose individuos cultivados de P. patula y Guadua angustifolia Kunth y especies dispersadas por aves y murciélagos, como Psidium guajava L. y Carica papaya L. En bosque, dos de las especies con mayor IVI son plantas cultivadas, Coffea arabica L. y Syzygium jambos (L.) Alston. La primera especie es un vestigio de la zona cafetera, mientras que la segunda es considerada invasora dispersada por el agua, aves y mamíferos (CABI., 2015) de la que, si bien fue muy abundante en el sotobosque, no se registraron individuos con DAP superior a $5 \mathrm{~cm}$. Por otro lado, en los pastizales prevalece la especie introducida $U$. decumbens.

El matorral se encuentra en un estado de colonización en el cual hay elementos de zonas abiertas como las especies herbáceas de Asteraceae y especies de bosque secundario como los árboles de $E$. citrifolium y E. cf. egensis. Además, en el estrato bajo del matorral se encontraron muchas especies compartidas con el bosque. Es decir, que el matorral está en proceso de sucesión vegetal y proporciona la sombra necesaria para que especies pioneras intermedias se establezcan.

De las especies pioneras intermedias registradas en este estudio, la mayoría suelen ser dispersadas por aves, las cuales cumplen un papel importante en la regeneración de estos bosques jóvenes. Esto se puede constatar en la alta colonización del sotobosque por plántulas de especies como $E$. cf. egensis, E. citrifolium y L. aggregatum (tabla 3), cuyos frutos son consumidos por aves. En contraste, especies más longevas como Pouteria torta (Mart.) Radlk., Aniba sp., Gustavia speciosa (Kunth) DC.,
Tapirira guianensis Aubl., Guatteria cargadero Triana \& Planch., Guarea sp., Trichilia pallida Sw. y Andira sp. son, en muchos casos, dispersadas por gravedad o por mamíferos y fueron muy escasas o ausentes en las parcelas, mientras que pueden ser más comunes en bosques maduros aledaños.

El banco de semillas de las especies de edades avanzadas o esciófitas en los fragmentos de bosque pudo ser afectado por la degradación del hábitat ocasionado por las actividades agropecuarias prolongadas en el tiempo, la deforestación y la tala selectiva de los árboles longevos. Adicionalmente, la distancia que existe entre los fragmentos de bosque secundario y bosque maduro limita la dispersión de semillas grandes. Por lo tanto, la lluvia de semillas la realizan en su mayoría animales voladores como aves y murciélagos, que por lo general consumen frutos de semillas pequeñas (e.g. frutos de plantas pioneras). Como consecuencia, la trayectoria sucesional hacia los bosques premontanos maduros tiende a ser muy lenta. Por esa razón, se recomienda realizar una intervención con programas de restauración a escala de poblaciones con el fin de repoblar los fragmentos de bosque secundario con estas especies de árboles longevos característicos de bosques premontanos para, de esta forma, recuperar las propiedades del ecosistema original.

\section{CONCLUSIONES}

El paisaje rural del piedemonte de los Farallones de Cali en la localidad de Loma Larga es un mosaico de pastizales, matorrales y bosques, resultado de la historia de uso del suelo que acompaña esta región. Se evidenció que el cambio de una matriz ganadera y agrícola a un proceso de regeneración natural por más de 20 años aumenta la diversidad vegetal en matorral y bosque, lo que muestra la resiliencia de este paisaje. El traslape de $50 \%$ de especies entre matorral y bosque muestra que estas comunidades están enriquecidas con especies de alta capacidad de dispersión. De hecho, 
los bosques son jóvenes, dominados por plantas pioneras intermedias nativas y ricos en plántulas de especies arbóreas en crecimiento, aunque no se registraron especies que pudieron existir en los bosques originales.

Si bien las especies de los tres elementos del paisaje juegan un papel importante en la regeneración natural de la vegetación y en el mantenimiento de diversas poblaciones vegetales y animales, la ausencia de propágulos de fases avanzadas retarda la trayectoria sucesional hacia el ecosistema original de bosque premontano. Es por eso que se recomienda realizar una intervención con programas de restauración con el fin de incorporar en el bosque especies de árboles longevos característicos de los bosques premontanos maduros. Las acciones que se emprendan desde ahora para conservar la diversidad existente y promover la restauración de las zonas degradadas permitirán mantener la heterogeneidad del paisaje y acelerar la recuperación de estos bosques.

\section{AGRADECIMIENTOS}

A Colciencias y a la Universidad del Valle por su apoyo con el programa Jóvenes Investigadores e Innovadores (Antonella Sardi). Al grupo de investigación de Ecología de Agroecosistemas y Hábitats Naturales (GEAHNA). A la Cooperativa Loma Larga por brindar su espacio. A Jorge Mario Ruiz, Fabio Nelson Ulabares y José Ricardo Vásquez por su ayuda en el trabajo de campo. A Pedro José Cardona, Alexander Vargas, María Esther Cardona y Carlos Parra por su ayuda en la identificación del material botánico. A Mercedes Andrade por su asesoría estadística. A Inge Armbrecht y William Beltrán por la revisión y comentarios del manuscrito.

\section{CONFLICTO DE INTERESES}

Los autores declaran no tener conflicto de intereses.

\section{CONTRIBUCIÓN POR AUTOR}

Los autores son los únicos responsables de la obra en todos los aspectos que condujeron a la elaboración de su publicación.

\section{REFERENCIAS BIBLIOGRÁFICAS}

Ariza, W., Toro, J. y Lores, A. (2009). Análisis florístico de los bosques premontanos en el municipio de Amalfi (Antioquia, Colombia). Colombia Forestal, 12(1), 81-102. DOI: https://doi.org/10.14483/udistrital.jour.colomb.for.2009.1.a07

Begon, M., Harper, J. y Townsend, C. (1999). Ecología, individuos, poblaciones y comunidades. Barcelona: Ediciones Omega.

Botina, J. y García, L. (2005). Árboles y arbustos del ecoparque Río Pance. Cali: Corporación Autónoma Regional del Valle del Cauca (CVC), Corporación para la Recreación Popular, Fondo para la Acción Ambiental.

Browning, D., Laliberte, A. y Rango, A. (2011). Temporal dynamics of shrub proliferation: linking parches to landscapes. International Journal of Geographical Information Science, 25(6), 913-930. DOI: https://doi.org/10.1080/13658816.2010.498789

Cantillo, E. y Fajardo, A. (2004). La reserva natural de Yotoco: su vegetación leñosa. Colombia Forestal, 8(1), 75-93. DOI: https://doi.org/10.14483/udistrital.jour.colomb.for.2004.1.a05

Cháves, C. y Rodríguez, S. A. (2012). Estructura y composición florística del bosque ribereño subandino de la subcuenca de Yumbillo, Yumbo (Valle del Cauca). Revista de Investigación Agraria y Ambiental, 3(1), 63-66. DOI: https://doi. org/10.22490/21456453.933

Corredor, G. (2016). Hábitat, su uso e historia natural del tinamí chico (Crypturellus soui) en el piedemonte de los Farallones de Cali, Colombia. Cali (tesis de doctorado). Doctorado en Ciencias-Biología, Facultad de Ciencias Naturales y Exactas, Universidad del Valle, Cali, Colombia. 
Cubiña, A. y Aide, M. (2001). The effect of distance from forest edge on seed rain and soil seed bank in a tropical pasture. Biotropica, 33(2), 260-267. DOI: https:// doi.org/10.1646/0006-3606(2001)033 [0260:TEODFF]2.0.CO;2

Denslow, J., Schultz, J., Vitousek, P. y Strain, B. (1990). Growth responses of tropical shrubs to treefall gap environments. Ecology, 71, 165-179. DOI: https:// doi.org/10.2307/1940257

Etter, A. y Wyngaarden, W. (2000). Patterns of landscape transformation in Colombia, with emphasis in the Andean Region. Ambio, 29(7), 432-439. DOI: https://doi.org/10.1579/0044-7447-29.7.432

Etter, A., McAlpine, C., Wilson, K., Phinn, S. y Possingham, H. (2006). Regional patterns of agricultural land use and deforestation in Colombia. Agriculture, Ecosystems and Environment, 114(2), 369-386. DOI: https://doi.org/10.1016/j.agee.2005.11.013

Fahrig, L. (2003). Effects of habitat fragmentation on biodiversity. Annual Review of Ecology, Evolution and Systematics, 34, 487-515. DOI: https://doi. org/10.1146/annurev.ecolsys.34.011802.132419

Gentry, A. (1982). Patterns of neotropical plant species diversity. Evolutionary Biology, 15, 1-84. DOI: https://doi.org/10.1007/978-1-4615-6968-8_1

Gentry, A. (1995). Patterns of diversity and floristic composition in neotropical montane forest. En S. B. Churchill, Biodiversity and Conservation of $\mathrm{Neo}-$ tropical Montane Forest (pp. 103-126). Nueva York: The New York Botanical Garden.

Giraldo-Castaño, D. (2000). Variación de la diversidad florística en un mosaico sucesional en la cordillera Central andina (Antioquia, Colombia). Darwiniana, 38(1-2), 33-42.

Hubbell, S. (1979). Tree dispersion, abundance and diversity in a Tropical Dry Forest. Science, 203, 1299-1309. DOI: https://doi.org/10.1126/ science.203.4387.1299

Hubbell, S. P., Foster, R. B. y O'Brien, S. T. (1999). Light gap disturbances recruitment limitation and tree diversity in a neotropical forest. Science, 283, 554-557. DOI: https://doi.org/10.1126/science.283.5401.554
Instituto de Hidrología, Meteorología y Estudios Ambientales (Ideam); Instituto Geográfico Agustín Codazzi (Igac); Instituto de Investigación de Recursos Biológicos Alexander von Humboldt (IAvH) (2007). Ecosistemas continentales, costeros y marinos de Colombia. Bogotá, D.C.: Ideam, Igac, IAvH, Instituto de Investigaciones Ambientales del Pacífico Jhon von Neumann.

Instituto de Hidrología, Meteorología y Estudios Ambientales (Ideam) (3 de junio de 2015). Datos meteorológicos en la estación de la Universidad del Valle de Cali. Recuperado de http://www.ideam. gov.co/

Jost, L.(2006). Entropyand diversity. Oikos, 363-375.DOI: https://doi.org/10.1111/j.2006.0030-1299.14714.x

Jost, L. (2007). Partitioning diversity into independent alpha and beta components. Ecology, 88, 24272439. DOI: https://doi.org/10.1890/06-1736.1

Kattán, G. y Naranjo, L. (2008). Regiones biodiversas. Herramientas para la planificación de sistemas regionales de áreas protegidas. Cali: Fundación Eco Andina, WCS y WWF.

Kuchler, A. (1966). Analyzing the physiognomy and structure of vegetation. Annals of the Association of American Geographers, 56, 112-127. DOI: https:// doi.org/10.1111/j.1467-8306.1966.tb00547.x

Laska, M. (1997). Structure of understory shrub assemblages in adjacent secondary and old growth tropical wet forest, Costa Rica. Biotropica, 19(1), 29-37. DOI: https://doi.org/10.1111/j.1744-7429.1997. tb00003.x

Londoño L, V. y Torres G. A. (2015). Estructura y composición vegetal de un bosque seco tropical en regeneración en Bataclán (Cali, Colombia). Colombia Forestal, 18(1), 71-85.

Lozano-Zambrano, F. H. (2009). Herramientas de manejo para la conservación de biodiversidad en paisajes rurales. Bogotá, D.C.: Instituto de Investigación de Recursos Biológicos Alexander von Humboldt y Corporación Autónoma Regional de Cundinamarca (CAR). 
Mayor, G., Gómez, L., Sarria, S., Cobo, A., Mejía, Y., Libreros, A., ... Mamiám, L. (2005). Plan de manejo 2005-2009 Parque Nacional Natural Farallones de Cali. Sistema de Parques Nacionales Naturales de Colombia. Cali: Embajada de los Países Bajos, Corporación Autónoma Regional del Valle del Cauca (CVC) y WWF.

Montaña, C. (1992). The colonization of bare areas in two-phase mosaics of an arid ecosystem. Journal of Ecology, 80(2), 315-327. DOI: https://doi. org/10.2307/2261014

R Core Team (2013). R: A language and environment for statistical computing. Vienna: R Foundation for Statistical Computing. Recuperado de http:// www.R-project.org/

Rangel, J. y Velásquez, A. (1997). Métodos de estudio de la vegetación. En J. Rangel, P. Lowey y M. Aguilar, Colombia- Diversidad Biótica II (pp. 17-47). Bogotá, D.C.: Universidad Nacional de Colombia-Instituto de Ciencias Naturales, Instituto de hidrología, Meteorología y Estudios Ambientales (Ideam)-Ministerio del Medio Ambiente, Comité de Investigaciones y Desarrollo Científico.

Reina, M., Medina, R., Ávila, F., Ángel, S. y Cortéz, R. (2010). Catálogo preliminar de la flora vascular de los bosques subandinos de la reserva biológica Cachalú, Santander (Colombia). Colombia Forestal, 13(1), 27-54. DOI: https://doi.org/10.14483/udistrital.jour.colomb.for.2010.1.a02

Robles, C. (2006). Caracterización de la diversidad y uso de la flora silvestre en el municipio de Donmatías, Antioquia. Medellín: Corporación Autónoma Regional del Centro de Antioquia.

Rodríguez, N., Armenteras, D., Morales, M. y Romero, M. (2006). Ecosistemas de los Andes colombianos. Bogotá, D.C.: Instituto de Investigación de Recursos Biológicos Alexander von Humboldt.

Rudas, G., Marcelo, D., Armenteras, D., Rodríguez, N., Morales, M., Delgado, L. y Sarmiento, A. (2007). Biodiversidad y actividad humana: relaciones en ecosistemas de bosque subandino en Colombia. Bogotá, D.C.: Instituto de Investigación de Recursos Biológicos Alexander von Humboldt.
Sarmiento, A., Galán, F. A., Mesa, C., Castaño, E., Delgado, C. L., y Ariza, F. (2002). Metodología de índices sintéticos de estado de los ecosistemas y relación con índices de presión y respuesta antrópica. Bogotá, D.C.: Programa Nacional de Desarrollo Humano- Departamento Nacional de Planeación, Instituto de Investigación de Recursos Biológicos Alexander von Humboldt.

Swaine, M. D. y Whitmore, T. C. (1988). On the definition of ecological species groups in tropical rain forests. Vegetation, 75, 81-86. DOI: https://doi. org/10.1007/BF00044629

Torres, A., Bautista, J., Cárdenas, M., Vargas, J., Londoño, V., Rivera, K., ... González, A. (2012). Dinámica sucesional de un fragmento de bosque seco tropical en el Valle del Cauca, Colombia. Biota Colombiana, 13(2), 66-85.

Trujillo, W. y Henao, M. (2018). Riqueza florística y recambio de especies en la vertiente orinoquense de los Andes. Colombia Forestal, 21(1), 18-33 DOI: https://doi.org/10.14483/2256201X.11848.

Vargas, W. (2012). Los bosques secos del Valle del Cauca, Colombia: una aproximación a su flora actual, Colombia. Biota Colombiana, 13(2), 102-164 DOI: https://doi.org/10.21068/bc.v13i2.265.

Vargas, W. (2015). Una breve descripción de la vegetación, con especial énfasis en las pioneras intermedias de los bosques secos de la Jagua, en la cuenca alta del río Magdalena en el Huila. Colombia Forestal, 18(1), 47-70. DOI: https://doi.org/10.14483/ udistrital.jour.colomb.for.2015.1.a03

Vásquez-Vélez, A. (2014). Estructura y diversidad de la vegetación del Parque Nacional Natural de la Isla Gorgona, Colombia. Revista Biología Tropical, 62(1), 13-26. DOI: https://doi.org/10.15517/rbt. v62i0.15976

Villarreal, H., M. Álvarez, S., Córdoba, F., Escobar, G., Fagua, F., Gast, H., ... Umaña, A. (2006). Manual de métodos para el desarrollo de inventarios de biodiversidad. Bogotá, D.C.: Programa de Inventarios de Biodiversidad. Instituto de Investigación de Recursos Biológicos Alexander von Humboldt. 
Anexo 1. Listado de especies de los elementos del paisaje de Loma larga, El Peón, Valle del Cauca, Colombia, entre abril y diciembre de 2013.

\begin{tabular}{|c|c|c|c|c|c|c|}
\hline \multirow[b]{2}{*}{ Familia } & \multirow[b]{2}{*}{ Especie } & \multicolumn{2}{|c|}{ Bosque } & \multicolumn{2}{|c|}{ Matorral } & \multirow[b]{2}{*}{ Pastizal } \\
\hline & & $\mathrm{DAP} \geq 5 \mathrm{~cm}$ & $\begin{array}{c}0.5<\mathrm{DAP} \leq \\
2.5 \mathrm{~cm}\end{array}$ & $\mathrm{DAP} \geq 5 \mathrm{~cm}$ & $\begin{array}{c}0.5<\text { DAP } \leq \\
25 \mathrm{~cm}\end{array}$ & \\
\hline Acanthaceae & Blechum sp. & & & & & $\mathrm{X}$ \\
\hline Araceae & Philodendron barrosoanum & & $\mathrm{x}$ & & $\mathrm{x}$ & \\
\hline Araliaceae & Dendropanax arboreus & $\mathrm{x}$ & $x$ & $\mathrm{x}$ & & \\
\hline Araliaceae & Schefflera morototoni & $x$ & $x$ & $x$ & $\mathrm{x}$ & \\
\hline Arecaceae & Chamaedorea tepejilote & & $x$ & & & \\
\hline Arecaceae & Prestoea acuminata & $\mathrm{x}$ & $\mathbf{x}$ & & & \\
\hline Asteraceae & Bidens sp. & & & & & $\mathrm{x}$ \\
\hline Asteraceae & Calea jamaicensis & & $x$ & & & \\
\hline Asteraceae & Chromolaena laevigata & & & & $x$ & $x$ \\
\hline Asteraceae & Chromolaena odorata & & & & & $x$ \\
\hline Asteraceae & Chromolaena sp. & & & & & $x$ \\
\hline Asteraceae & Chromolaena sp. 2 & & & & & $x$ \\
\hline Asteraceae & Chromolaena tacotana & & & & $x$ & \\
\hline Asteraceae & Delilia sp. & & & & & $\mathrm{X}$ \\
\hline Asteraceae & Elephantopus mollis & & & & & $\mathrm{x}$ \\
\hline Asteraceae & Eleutheranthera sp. & & & & & $x$ \\
\hline Asteraceae & Emilia fosbergii & & & & $x$ & $x$ \\
\hline Asteraceae & Eupatorium sp. & & & & & $x$ \\
\hline Asteraceae & Indeterminada & & & & $x$ & \\
\hline Asteraceae & Indeterminada & & & & $x$ & \\
\hline Asteraceae & Indeterminada & & & & $\mathrm{X}$ & \\
\hline Asteraceae & Indeterminada & & & & $\mathrm{x}$ & \\
\hline Asteraceae & Indeterminada & & & & $x$ & \\
\hline Asteraceae & Indeterminada & & & & & $x$ \\
\hline Asteraceae & Mikania sp. & & $x$ & & $x$ & $x$ \\
\hline Asteraceae & Pseudelephantopus spiralis & & & & & $\mathrm{X}$ \\
\hline Bignoniaceae & Indeterminada & $x$ & & & & \\
\hline Caricaceae & Carica papaya & & & & $x$ & \\
\hline Clusiaceae & Clusia minor & $x$ & $x$ & $x$ & $X$ & $x$ \\
\hline Clusiaceae & Clusia palmicida & $x$ & $x$ & $x$ & & \\
\hline Commelinaceae & Dichorisandra hexandra & & $\mathrm{x}$ & & & \\
\hline Commelinaceae & Commelina obliqua & & $x$ & & & \\
\hline Convolvulaceae & Indeterminada & & & & & $x$ \\
\hline Costaceae & Costus chartaceus & & $\mathrm{x}$ & & & \\
\hline
\end{tabular}




\begin{tabular}{|c|c|c|c|c|c|c|}
\hline \multirow[b]{2}{*}{ Familia } & \multirow[b]{2}{*}{ Especie } & \multicolumn{2}{|c|}{ Bosque } & \multicolumn{2}{|c|}{ Matorral } & \multirow[b]{2}{*}{ Pastizal } \\
\hline & & DAP $\geq 5 \mathrm{~cm}$ & $\begin{array}{c}0.5<\text { DAP } \leq \\
2.5 \mathrm{~cm}\end{array}$ & $\mathrm{DAP} \geq 5 \mathrm{~cm}$ & $\begin{array}{c}0.5<\text { DAP } \leq \\
25 \mathrm{~cm}\end{array}$ & \\
\hline Cyperaceae & Cyperus sp. & & & & & $x$ \\
\hline Cyperaceae & Rhynchospora nervosa & & & & & $x$ \\
\hline Cyperaceae & Scleria melaleuca & & & & $x$ & \\
\hline Erythroxylaceae & Erythroxylum citrifolium & $x$ & $x$ & $X$ & $x$ & \\
\hline Euphorbiaceae & Alchornea latifolia & & $x$ & & & \\
\hline Euphorbiaceae & Chamaesyce sp. & & & & & $x$ \\
\hline Euphorbiaceae & Croton gossypiifolius & $x$ & & & $x$ & $x$ \\
\hline Euphorbiaceae & Croton sp. & & $x$ & & & \\
\hline Euphorbiaceae & Tetrorchidium rubrivenium & $x$ & $x$ & & & \\
\hline Fabaceae & Calliandra pittieri & $x$ & $x$ & & & \\
\hline Fabaceae & Desmodium sp. 1 & & & & & $x$ \\
\hline Fabaceae & Desmodium sp. 2 & & & & & $x$ \\
\hline Fabaceae & Desmodium sp. 3 & & & & & $x$ \\
\hline Fabaceae & Mimosa pudica & & & & & $x$ \\
\hline Fabaceae & Senna macrophylla & & & & $x$ & \\
\hline Fabaceae & Stylosanthes guianensis & & & & & $x$ \\
\hline Fabaceae & Vigna sp. & & & & & $x$ \\
\hline Indeterminada & & & & & & $x$ \\
\hline Indeterminada & & & & & & $x$ \\
\hline Indeterminada & & & & & & $x$ \\
\hline Lacistemataceae & Lacistema aggregatum & $x$ & $x$ & $x$ & $x$ & \\
\hline Lamiaceae & Indeterminada & & & & & $x$ \\
\hline Lauraceae & Cinnamomum triplinerve & $\mathrm{x}$ & $x$ & $x$ & $x$ & \\
\hline Lauraceae & Nectandra acutifolia & $x$ & $x$ & & & \\
\hline Lauraceae & Nectandra sp. 1 & $x$ & $x$ & & & \\
\hline Lauraceae & Ocotea aurantiodora & $x$ & $x$ & $x$ & $x$ & $x$ \\
\hline Lauraceae & Ocotea sp. & $x$ & & & & \\
\hline Lauraceae & Persea caerulea & & & $x$ & $x$ & \\
\hline Malpighiaceae & Hiraea fagifolia & & $x$ & & & \\
\hline Malvaceae & Sida sp. & & & & & $x$ \\
\hline Malvaceae & Triumfetta sp. & & & & & $x$ \\
\hline Melastomataceae & Clidemia ciliata & & $x$ & & $x$ & $x$ \\
\hline Melastomataceae & Henriettea seemannii & $x$ & $x$ & $x$ & $x$ & \\
\hline Melastomataceae & Miconia albicans & & & & $x$ & \\
\hline Melastomataceae & Miconia caudata & & & $x$ & & \\
\hline Melastomataceae & Miconia dodecandra & & & & $x$ & \\
\hline Melastomataceae & Miconia macrotis & & $x$ & & $x$ & \\
\hline
\end{tabular}




\begin{tabular}{|c|c|c|c|c|c|c|}
\hline \multirow[b]{2}{*}{ Familia } & \multirow[b]{2}{*}{ Especie } & \multicolumn{2}{|c|}{ Bosque } & \multicolumn{2}{|c|}{ Matorral } & \multirow[b]{2}{*}{ Pastizal } \\
\hline & & $\mathrm{DAP} \geq 5 \mathrm{~cm}$ & $\begin{array}{c}0.5<\text { DAP } \leq \\
2.5 \mathrm{~cm}\end{array}$ & $\mathrm{DAP} \geq 5 \mathrm{~cm}$ & $\begin{array}{c}0.5<\text { DAP } \leq \\
25 \mathrm{~cm}\end{array}$ & \\
\hline Melastomataceae & Miconia matthaei & $\mathrm{x}$ & $x$ & X & $x$ & \\
\hline Melastomataceae & Miconia minutiflora & $x$ & $x$ & $x$ & $x$ & \\
\hline Melastomataceae & Miconia rubiginosa & & & $x$ & $x$ & \\
\hline Melastomataceae & Miconia stenostachya & & & & $x$ & $x$ \\
\hline Melastomataceae & Miconia trinervia & & & $x$ & $x$ & \\
\hline Melastomataceae & Miconia sp. & & & & $x$ & \\
\hline Melastomataceae & Miconia sp. 2 & & & & & $x$ \\
\hline Meliaceae & Guarea guidonia & $x$ & & & & \\
\hline Meliaceae & Trichilia pallida & $x$ & $x$ & & & \\
\hline Meliaceae & Indeterminada & & $x$ & & & \\
\hline Myrtaceae & Eugenia cf. egensis & $\mathrm{x}$ & $x$ & $x$ & $x$ & \\
\hline Myrtaceae & Myrcia popayanensis & $x$ & $x$ & $x$ & $x$ & \\
\hline Myrtaceae & Psidium guajava & & & $x$ & $x$ & \\
\hline Myrtaceae & Psidium guineense & & & & $x$ & \\
\hline Myrtaceae & Syzygium jambos & & $x$ & & $x$ & \\
\hline Orchidaceae & Galeandra beyrichii & & $x$ & & & \\
\hline Pinaceae & Pinus patula & & & $x$ & & \\
\hline Piperaceae & Piper aduncum & & $x$ & & $x$ & \\
\hline Piperaceae & Piper augustum & & $x$ & & & \\
\hline Piperaceae & Piper crassinervium & & $x$ & & $x$ & \\
\hline Piperaceae & Piper reticulatum & & $x$ & & $x$ & \\
\hline Piperaceae & Piper sp. 1 & & $x$ & & $x$ & \\
\hline Piperaceae & Piper sp. 2 & & $x$ & & & \\
\hline Plantaginaceae & Angelonia angustifolia & & & & $x$ & $x$ \\
\hline Poaceae & Andropogon sp. & & & & & $x$ \\
\hline Poaceae & Digitaria sp. & & & & & $x$ \\
\hline Poaceae & Guadua angustifolia & & $x$ & $x$ & $x$ & \\
\hline Poaceae & Gynerium sagittatum & & $x$ & & & \\
\hline Poaceae & Homolepis aturensis & & & & & $x$ \\
\hline Poaceae & Hyparrhenia rufa & & & & & $x$ \\
\hline Poaceae & Indeterminada & & & & & $x$ \\
\hline Poaceae & Indeterminada & & & & & $x$ \\
\hline Poaceae & Indeterminada & & & $x$ & & \\
\hline Poaceae & Ischaemum sp. & & & & $x$ & \\
\hline Poaceae & Olyra latifolia & & $x$ & & $x$ & \\
\hline Poaceae & Oplismenus sp. & & & & & $x$ \\
\hline Poaceae & Steinchisma laxum & & & & & $x$ \\
\hline
\end{tabular}




\begin{tabular}{|c|c|c|c|c|c|c|}
\hline \multirow[b]{2}{*}{ Familia } & \multirow[b]{2}{*}{ Especie } & \multicolumn{2}{|c|}{ Bosque } & \multicolumn{2}{|c|}{ Matorral } & \multirow[b]{2}{*}{ Pastizal } \\
\hline & & $\mathrm{DAP} \geq 5 \mathrm{~cm}$ & $\begin{array}{c}0.5<\text { DAP } \leq \\
2.5 \mathrm{~cm}\end{array}$ & $\mathrm{DAP} \geq 5 \mathrm{~cm}$ & $\begin{array}{c}0.5<\text { DAP } \leq \\
25 \mathrm{~cm}\end{array}$ & \\
\hline Poaceae & Setaria parviflora & & & & & $x$ \\
\hline Poaceae & Urochloa decumbens & & & & & $x$ \\
\hline Polygalaceae & Polygala sp. & & & & & $x$ \\
\hline Polygonaceae & Polygonum sp. & & & & $x$ & \\
\hline Primulaceae & Myrsine pellucida & $x$ & $x$ & $x$ & $x$ & \\
\hline Primulaceae & Parathesis candolleana & & $x$ & & & \\
\hline Rubiaceae & Chiococca alba & & $x$ & & & \\
\hline Rubiaceae & Coccocypselum lanceolatum & & & & & $x$ \\
\hline Rubiaceae & Coffea arabica & & $x$ & & & \\
\hline Rubiaceae & Geophila macropoda & & & & & $x$ \\
\hline Rubiaceae & Ladenbergia oblongifolia & $x$ & $x$ & $\mathrm{x}$ & $x$ & \\
\hline Rubiaceae & Psychotria micrantha & & $x$ & & & \\
\hline Rubiaceae & Psychotria racemosa & & $x$ & & & \\
\hline Rubiaceae & Psychotria deflexa & & $x$ & & $x$ & \\
\hline Rubiaceae & Spermacoce sp. & & & & & $x$ \\
\hline Salicaceae & Casearia sylvestris & $x$ & & & $x$ & \\
\hline Sapindaceae & Allophylus racemosus & $\mathrm{x}$ & $x$ & & & \\
\hline Sapindaceae & Cardiospermum corindum & & $x$ & & & \\
\hline Sapindaceae & Cupania americana & $x$ & $x$ & & $x$ & $x$ \\
\hline Sapindaceae & Paullinia faginea & & $x$ & & & \\
\hline Sapotaceae & Pouteria torta & & $x$ & & & \\
\hline Siparunaceae & Siparuna laurifolia & & $\mathrm{x}$ & & $x$ & \\
\hline Solanaceae & Cestrum nocturnum & $x$ & & & & \\
\hline Solanaceae & Solanum lepidotum & & $x$ & & & \\
\hline Solanaceae & Solanum pseudolulo & & & & & $x$ \\
\hline Urticaceae & Cecropia angustifolia & $x$ & & & & \\
\hline Urticaceae & Urera baccifera & & $x$ & & & \\
\hline Verbenaceae & Petrea pubescens & $x$ & $x$ & $x$ & & \\
\hline Verbenaceae & Lantana camara & & & & $x$ & \\
\hline Verbenaceae & Indeterminada & & & & $x$ & \\
\hline Violaceae & Indeterminada & $x$ & & & & \\
\hline
\end{tabular}

